\title{
Mobile Based Leaf Disease Classifier
}

\author{
Chandrakala D', Sarath Kishore R², Kishore R3, Nandha Kumar M. $K^{4}$ \\ ${ }^{1}$ Professor, Department of Computer Science and Engineering, \\ ${ }^{2,3,4}$ Student, Department of Computer Science and Engineering, \\ Kumaraguru College of Technology, Coimbatore, Tamil Nadu , India
}

\section{ABSTRACT}

Crop diseases pose a significant threat to food security and yield, but their rapid and efficient identification is still a complicated and cumbersome process in many parts of the world because of the lack of related infrastructure. The advent of A.I., current advances in the areas of image processing, and the increasing dispersion of mobile devices into the masses, advocates the idea of mobile-based disease identification. Using a public dataset of over 20000 images of healthy and infected tomato, potato leaves that were collected under certain controlled conditions, a deep Convolutional Neural Network (CNN) has been trained to analyze and identify 15 diseases of the said leaves. The trained model achieved an accuracy of $89.325 \%$ on a test set, illustrating the feasibility of this method. Overall, this method of training diverse deep learning data models on progressively large and diverse image datasets lays a clear foundation for mobile-based crop disease identification on a massive scale.

KEY WORDS: DISEASE CLASSIFIER, CONVOLUTION NEURAL NETWORK, VANISHING GRADIENT, NORMALIZATION, POOLING.

\section{INTRODUCTION}

Human life has largely depended on edible crops to survive, for a long time. Modern technologies have enabled human society to produce enough food crops to meet the demand of the growing population of 7 billion and maintain sustainability. However, there is still a major threat to food security owing to several factors such as pest infestation, plant diseases, fall in pollination, climate changes. Plant diseases are a major threat not only for food sustainability but also for small scale farmers and other such people whose livelihood depends mainly on healthy crops and about $80 \%$ of the agricultural production is generated by the likes of such people.

\section{ARTICLE INFORMATION}

*Corresponding Author: chandrakala.d.cse@kct.ac.in Received 25th Oct 2020 Accepted after revision 9th Dec 2020 Print ISSN: 0974-6455 Online ISSN: 2321-4007 CODEN: BBRCBA

Thomson Reuters ISI Web of Science Clarivate Analytics USA and Crossref Indexed Journal

\section{Clarivate crossef}

NAAS Journal Score 2020 (4.31) SJIF: 2020 (7.728)

A Society of Science and Nature Publication,

Bhopal India 2020. All rights reserved.

Online Contents Available at: http//www.bbrc.in/

Doi: http://dx.doi.org/10.21786/bbrc/13.11/19
Various methods are put in place to prevent crop loss due to pests and diseases. Traditional methods include the widespread application of pesticides. But it is essential to identify the disease correctly when it appears in order to apply the appropriate pesticide. It is a crucial step in efficient disease management. Historically, this is done by agricultural organizations and universities and other such institutions wherein the same is sent to the concerned expert who analyses the sample and determines the route of remedy.

In recent times, with the advent of the Internet, such traditional efforts are additionally supported and by the instant provision of relevant information aiding to an online diagnosis process. There are billions of mobile devices in use among the masses today, further advocating the processes related to an online solution. Smartphones in particular offer a variety of new approaches with their efficient computing power, highresolution cameras and displays, a wide array of sensors and accessories prebuilt into them. All these features packed together make it plausible for a situation where

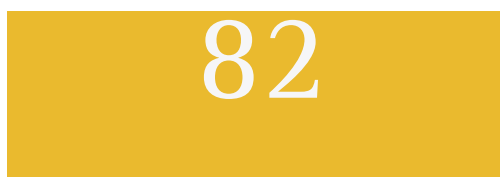


image-based disease diagnosis based on neural networks can be made available on an unprecedented scale. Here, the feasibility of using the Convolutional Neural Network (CNN) approach utilizing 22,953 images of 3 crop species with 15 diseases and healthy leaves from the publicly available Plant Village dataset is established.

The Large-Scale Visual Recognition Challenge (ILSVRC) which is based on the ImageNet dataset has been widely used as benchmarks for numerous problems in computer vision including object recognition. Following the top-5 error rate of $16.4 \%$ achieved by a deep convolutional neural network for classification of 1000 categories, many advances have been made in the field of deep convolutional networks which has significantly reduced the error. Training of large neural networks on huge datasets is very time-consuming and requires heavy computing power. But once these models are trained, they can be deployed anywhere. The trained models can classify the images quickly and do not require much computing requirements, making it very much suitable for smartphones. To make accurate classifications, the model must be trained over a diverse and large dataset. This problem was solved by the Plant Village dataset project where they collected tens of thousands of images of healthy and diseased crops of various species. Here, a deep convolutional model that classifies 15 diseases in 3 crop species has been trained.

The best performing model achieved an accuracy of 89.325\% thus advocating the feasibility of this approach. Vaijinath B et. al. implemented leaf disease detection using image processing and Support Vector Machine (SVM), the input image was first blur softened to reduce the noise (Vaijinath B Batule, Gaurav U Chavan, Vishal P Sanap, Kiran D Wadka, 2016). Then the image was converted from RGB (Red, Green, Blue) to HSV (Hue, Saturation, Intensity) for efficient color thresholding. Then separation of foreground and background was done for leaf segmentation from the image. After the image segmentation feature extraction was performed and given as input to the SVM and $\mathrm{K}$-means algorithm to get the desired results.

Belal A M Ashqar and Samy S. Abu-Naser presented an Image-Based Tomato Leaves Diseases Detection Using Deep Learning. (Ashqar, Belal, Abu-Naser Samy, 2018). They used tomato leaf images of 5 different disease categories from the plant village dataset namely Bacterial Spot, Early Blight, Healthy, Septorial Leaf Spot, Leaf mold, Yellow Leaf Curl Virus. They implemented a Deep learning technique and arrived with an accuracy of 99.84\% for full color and 95.54\% for grayscale model. Drjoy Sen Maitra et. al. presented a CNN based approach to handwritten character recognition for multiple scripts of India. (Sen Maitra Durjoy, Bhattacharya, Ujjwal, Parui, Swapan. 2015).

They used 6 datasets of 100 classes namely Bangla basic characters, Bangla numerals, Devanagari numerals, Oriya numerals, Telugu numerals, English (MNIST - Modified National Institute of Standards and Technology database) numerals. The images were converted into grayscale and trained for more than 1000 iteration to get an accuracy of 85\% and used the obtained feature as an input to the SVM model and the desired output was produced where the accuracy of Bangla basic characters, Bangla numerals, Devanagari numerals, Oriya numerals, Telugu numerals, English (MNIST) numerals are 95.6\%, 98.37\%, 98.54, 97.2\%, 96.5\%, and 99.10\% respectively.

Sabah Bashir and Navdeep Sharma presented an image processing technique to detect diseases in Malus Domestica using image processing in MATLAB (Sabah Bashir, Navdeep Sharma, 2012). The image was taken in RGB format to facilitate the creation of a histogram for the comparison of images. K-means clustering technique was used for the classification of images based on disease. Then threshold separation of the image was done to separate the background and foreground. Sujatha R et. al. presented a technique to identify diseases in the leaf efficiently (Sujatha R, Sravan Kumar Y, Garine Uma Akhil 2017). Using the K-means clustering image was segmented into $\mathrm{K}$ groups representing segments of the image and SVM technique used to distinguish the disease and hence arrive at the desired results. Prajakta Mitkal et. al. presented an image processing technique for the detection of sugarcane leaf diseases (Prajakta Mitkal, Priyanka Pawar, Mira Nagane, Priyanka Bhosale, Mira Padwal and Priti Nagane, 2016). Authors used SVM, NonLinear SVM, and Multiclass SVM for feature extractions and obtained high accuracy.

Figure 1: Proposed Methodology

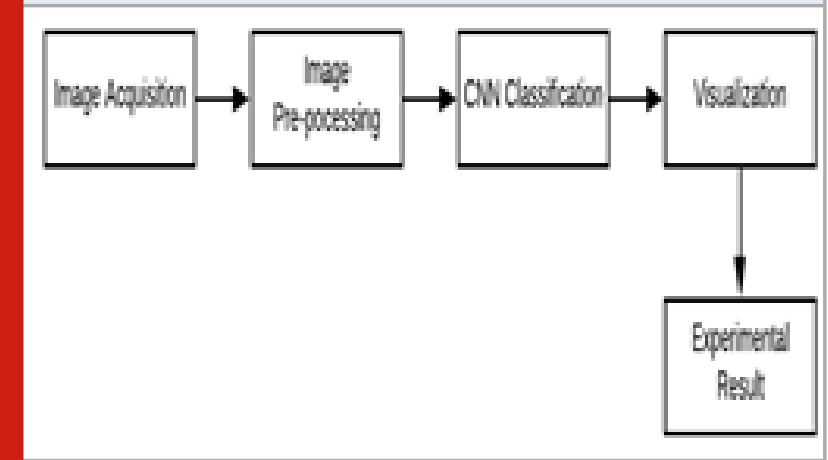

Prakash M Manikar et. al. described a plant disease recognition technique which uses the Spatial Gray-level Dependence Matrices (SGDM) and K-means clustering (Prakash M. Mainkar, Shreekant Ghorpade,, Mayur Adawadkar, 2015). K-means technique is used to segment the image. The infected clusters are converted from RGB color space to HSI and the SGDM matrix was generated for Hue and Saturation. In the next phase, the GLCM function was used to calculate the features and compute the texture statistics. In the last phase, extracted features were passed through a neural network that is trained for disease recognition. Ghulam Mustafa Choudhary and Vikrant Gulati presented an image processing technique for the detection of Scorch and Spot diseases of plants (Ghulam Mustafa Choudhary, Vikrant Gulati, 2015). Firstly, the leaf image is acquired in the RGB color format. 
Then a color transformation structure was created and the RGB color values are converted to that space in the pre-processing. K-means clustering was applied, and green pixels were masked to remove those cells inside the boundaries of infected clusters and obtain useful segments of the image. The color co-occurrence method was used to compute color, texture, and edge features. Lastly, the disease was classified by a configured neural network.

Hrishikesh P. Kanjalkar and S.S.Lokhande explained how feature extraction is useful in simplifying the computation power and memory size due to a combination of variables (Hrishikesh P. Kanjalkar, S.S.Lokhande, 2014). In this work, the leaf spots are segmented from the leaves using the application of graph theory algorithm. Pradnya Ravindra Narvekar et. al. identified the disease in the grape leaf by making use of image processing techniques (Pradnya Ravindra Narvekar, Mahesh Manik Kumbhar, S N Patil, 2014). Distance between the concurrent spots was identified and the distance was calculated. Features considered in this method are color and texture of the image. This method calculates two values such as cluster shade and cluster prominence, by comparing these values the disease can be identified. Vijai Singh and Mishra detected a plant infection using image processing techniques (Vijai Singh,, A. K. Mishra 2017). The infections considered here are Anthracnose, Cercospora leaf spot, and bacterial blight. The images were either captured from the mobile or retrieved from mobile storage. From the texture values obtained from the Gray Level Co-occurrence Matrix (GLCM), the disease was classified. Ghulam Mustafa Choudhary extracted features such as color, texture, morphology, and structure by using the GLCM method. The model was built using Support Vector Machine to classify the diseases.

Sharada Prasanna Mohanty experimented deep learning for the detection of plant disease (Sharada Prasanna Mohanty 2016). The deep learning architecture involved were AlexNet and GoogLeNet. The dataset involved in the study contained 54,306 consisting of 38 classes (14 crop species and 26 diseases). For interpreting the model with more accuracy, varying configurations were used by changing the color space representation and training and testing set distribution. The color space representation consisted of color, grayscale, and leaf segmented, and the training-testing distribution as 80\%20\%, 60\%-40\%, and 50\%-50\%. The highest accuracy level obtained by varying the configuration was $98.36 \%$, the configurations used were GoogLeNet, Color and 80\%$20 \%$ distribution ratio.

\section{METHODOLOGY}

Convolution neural networks is a part of deep neural networks, which makes the algorithm to precisely identify the deeper level of the image as a composition of edges, curves, line, and other contents, instead of just going through the image roughly like in other neural networks. CNN is inspired by the visual cortex of a human brain, which has certain regions of cells specific to certain regions of the visual field. Features of the image(filters) that have to be manually fed to classify an image in traditional image classification techniques were made to learn by itself on CNN so it drastically reduced the human effort of feeding it. An input of image is passed through hidden layers namely convolutional, nonlinear, pooling (downsampling), and fully connected layers, and get an output which can be a single class or a probability of classes that best describes the image. The purpose of this layer is to extract the features of the image that describe the input image and it is the first layer of CNN. Here a filter size is defined which is based on, how detailed the precision of feature recognition, smaller the filter matrix higher the precision.

A filter is taken and multiplied with the image matrix from the top and traversed column wise followed by rows. As a result, a feature map that identifies the curves, edges, lines, or features of the image is obtained. The region of the image matrix that is multiplied with is called the receptive field. Padding is used to augment the shrunken feature map to the original size of the image or to make it bigger than the original image. This must be done to avoid getting a reduced feature map that is $2 \times 2$ matrix after each convolution layer. Activation layer essentially follows each convolution layer. When the system is working on linear operations during Conv layers, activation function is added to bring non-linearity to the function.

Vanishing gradient problem is the issue where the gradient decreases exponentially from layer to layer, which also causes the lower layer to train slowly and this issue is solved by ReLU. The function $\mathrm{f}(\mathrm{x})=\max (0, \mathrm{x})$ is applied to all the ReLU layers to all of the values from the input to this layer. ReLU turns all the negative values to zero. This layer increases the nonlinear properties of the model and the overall network without affecting the receptive fields of the conv layer.

Pooling is used to reduce the image size in terms of width and height. It is of two types, max-pooling which reduces the image size by taking the largest value in that window and average pooling which reduces the image size by taking the average value in that window. Dropout is used to drop a random activation function by setting it to zero which prevents the model from being overfitted to the training set. The model should even be able to identify and classify images based on feature. It is used to convert the pooled feature map into a sequential column of numbers like a long vector. Then this is used as an input layer of an artificial neural network for further processing. The first step is to acquire images of various layers CNN is used to extract features and classify them based on the feature map. Figure 1 shows the flow of the process of proposed methodology. The flow of the process of proposed system is: (i) Image Acquisition (ii) Image Preprocessing (iii) CNN Classifier (iv) Visualization (v) Experimental Result.

Image Acquisition: The dataset is obtained from the public Plant Village project database, which contains 
22,953 images of 3 crop species and 12 diseases. This database consists of 15309 images of Tomato leaves, 4668 images of Potato leaves, and 2974 images of Pepper Bell. This dataset has samples of healthy leaves in addition to diseased leaves. https://ww.kaggle.com/ emmarex/plantdisease

It has 15 classes in total as follows:

- Class(0): Tomato Target Spot'

- Class(1): Tomato Mosaic Virus

- Class(2): Pepper Bell Bacterial Spot

- Class(3): Pepper Bell Healthy

- Class(4): Potato Early Blight

- Class(5): Potato Healthy

- Class(6): Potato Late Blight

- Class(7): Tomato Yellow Leaf Curl Virus

- Class(8): Tomato Bacterial Spot

- Class(9): Tomato Early Blight

- Class(10): Tomato Healthy

- Class(11): Tomato Late Blight

- Class(12): Tomato Leaf Mold

- Class(13): Tomato Septoria Leaf Spot

- Class(14): Tomato Spider Mites

Image Preprocessing: The acquired images are converted into a 256x256 image size. Due to differences in a number of images in each class of the dataset, image augmentations like rotation, width shift, height shift, brightness, shearing, horizontal flip, vertical flip are used to generate new images from existing datasets to maintain a standard number of images in each class, To achieve this ImageDataGenerator from Keras was used. Then the images are divided based on the train and test ratio and passed into the CNN classifier.

Figure 2: Dataset Samples
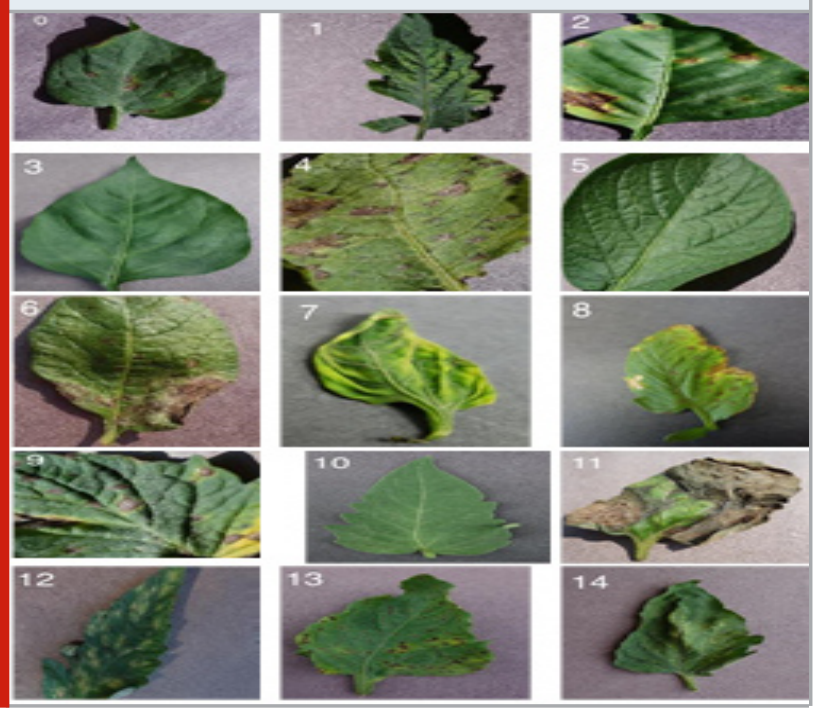

Classification: This model takes color images as input, so Convolutional Neural Networks (CNNs) are used to extract features from the leaf images. The resulting model has two parts: The first part of the model has Convolution layers with ReLU activation function, Normalization layers, Max Pooling layers, and Dropout layers arranged in sets of different combinations. The second part of the model is the set of Dense and Flatten layers combined with a Normalization and Dropout layers followed by an individual Dense layer with SoftMax activation function and 15 outputs representing the 15 classes.In the Figure 3 , CNN architecture of our model is represented and some acronyms used in the figure are C - conv2d, R - Relu, $\mathrm{B}$ - batch normalizer, MP - max pooling, DO - drop out Visualization: To understand how the model works and what is exactly being learned, the intermediate layer activations that display the various feature maps output by various Convolution, Normalization, and Max Pooling layers in a network when a certain input is given are visualized. This helps to get an idea of how the input image is decomposed into different filters by the network. Figure 4,5,6 shows the various layers outputs.

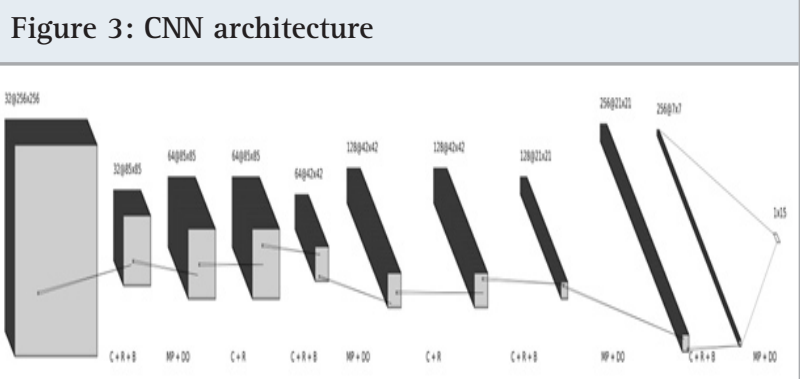

Table 1. Model Summary

\begin{tabular}{|c|c|c|c|}
\hline Layex (type) & Output & Shape & Param \# \\
\hline conv2d_1 (Conv2D) & (None, & $256,256,321$ & 896 \\
\hline activation_1 (Activation) & iNone. & $256,256,321$ & 0 \\
\hline batch_normalization_1 (Batch & (None, & $256,256,321$ & 128 \\
\hline max_pooling2d_1 (MaxPooling2 & (None. & $85,85,321$ & o \\
\hline dropout_1 (Dropout) & iNone, & $85,85,321$ & 0 \\
\hline conv2d_2 (Conv2D) & iNone, & $85,85,64)$ & 18496 \\
\hline activation_2 (Activation) & iNone. & $85,85,64)$ & o \\
\hline Conv2d_3 (Conv2D) & (None. & $85,85,641$ & 36928 \\
\hline activation_3 (Activation) & iNone. & $85,85,641$ & o \\
\hline batch_normalization_2 (Batch & (None. & $85,85,64)$ & 256 \\
\hline max_pooling2d_2 (MaxPooling2 & (None. & $42,42,641$ & o \\
\hline dropout_2 (Dropout) & (None. & $42,42,641$ & 0 \\
\hline Conv2d_4 (Conv2D) & iNone, & $42,42,128)$ & 73856 \\
\hline activation_4 (Activation) & (None. & $42,42,128)$ & 0 \\
\hline conv2d_5 (Conv2D) & (None. & $42,42,128)$ & 147584 \\
\hline activation_5 (Activation) & iNone. & $42,42,128)$ & o \\
\hline batch_normalization_ ${ }^{3}$ (Batch & (None. & $42,42,128)$ & 512 \\
\hline max_pooling2d_3 (MaxPooling2 & (None. & $21,21,128)$ & o \\
\hline dropout_3 (Dropout) & (None. & $21,21,128)$ & 0 \\
\hline Conv2d_6 (Conv2D) & (None, & $21,21,256)$ & 295168 \\
\hline activation_6 (Activation) & (None, & $21,21,256)$ & 0 \\
\hline batch_normalization_4 (Batch & (None, & $21,21,256)$ & 1024 \\
\hline max_pooling2d_4 (MaxPooling2 & (None, & $7,7,256)$ & 0 \\
\hline dropout_4 (Dropout) & (None, & $7,7,256)$ & 0 \\
\hline flatten_1 (Flatten) & (None, & $12544)$ & 0 \\
\hline dense_1 (Dense) & (None, & 1024) & 12846080 \\
\hline activation_7 (Activation) & (None, & 1024) & 0 \\
\hline batch_normalization_5 (Batch & (None, & 1024) & 4096 \\
\hline dropout_5 (Dropout) & (None, & 1024) & 0 \\
\hline dense_2 (Dense) & (None, & 15) & 15375 \\
\hline activation_8 (Activation) & (None, & 15) & 0 \\
\hline
\end{tabular}


Figure 4: Full-Color intermediate activation in the Convolution layer

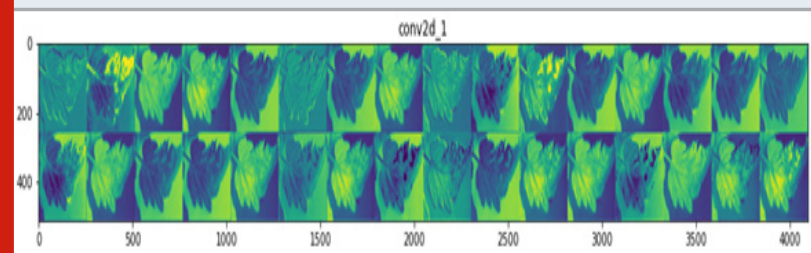

Figure 5: Full-Color intermediate activation in Pooling layer

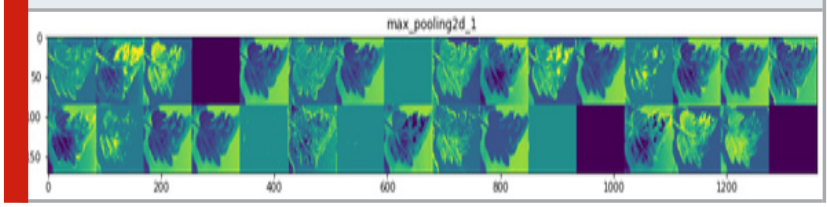

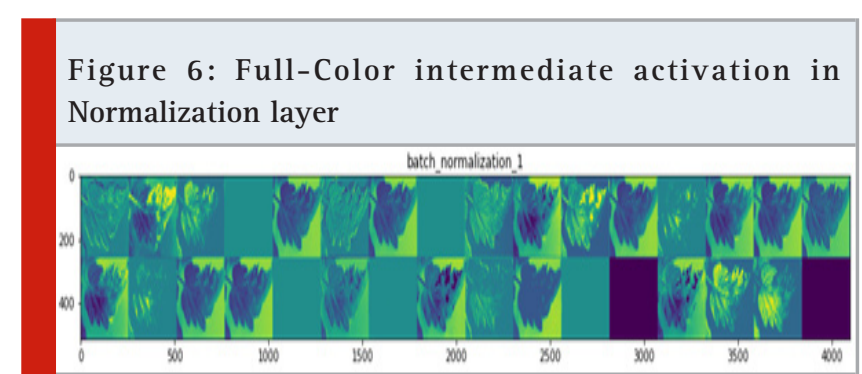

Table 2. Experimental Result

\begin{tabular}{|l|c|c|c|c|c|c|}
\hline $\begin{array}{l}\text { Activation } \\
\text { Function }\end{array}$ & Optimizer & $\begin{array}{c}\text { Learning } \\
\text { Rate }\end{array}$ & $\begin{array}{c}\text { \# of } \\
\text { Epochs }\end{array}$ & $\begin{array}{c}\text { Batch } \\
\text { Size }\end{array}$ & Loss & $\begin{array}{c}\text { Accuracy } \\
\text { in \% }\end{array}$ \\
\hline Relu & adadelta(rho=0.95) & 1.0 & 20 & 64 & 1.3584 & 62.70 \\
\hline Relu & adadelta(rho=0.95) & 1.0 & 10 & 32 & 0.0446 & 89.32 \\
\hline Relu & adadelta(rho=0.95) & 1.0 & 10 & 32 & 0.3113 & 88.43 \\
\hline Relu & adadelta(rho=0.95) & 1.0 & 10 & 32 & 0.1344 & 87.17 \\
\hline Relu & adadelta(rho=0.95) & 1.0 & 9 & 32 & 0.0054 & 88.05 \\
\hline Relu & adadelta(rho=0.95) & 1.0 & 30 & 32 & 0.4531 & 87.12 \\
\hline Relu & adamax & 0.002 & 20 & 64 & 1.8768 & 62.10 \\
\hline Relu & Adam & 0.001 & 20 & 64 & 0.5292 & 84.54 \\
\hline Relu & Nadam & 0.002 & 20 & 64 & 0.9715 & 74.02 \\
\hline Relu & Nadam & 0.002 & 20 & 32 & 1.2239 & 71.76 \\
\hline Relu & Nadam & beta1=.9 beta2=.99 & & & & \\
\hline
\end{tabular}

\section{RESULTS}

Different CNN models with different attributes were trained and the model in [Table 1] yielded the better results. Once a proper model was obtained, learning parameters of that model was varied and different accuracies as tabulated in the Table 2. That model under the setting of Optimizer: adadelta (1.0, rho=0.95), Loss function: Categorical Cross Entropy, train dataset ratio of 70\% and test dataset ratio of 30\% yielded the best accuracy of $89.325 \%$ on full color images.

\section{CONCLUSION}

In this article, CNN based leaf disease detection method is proposed. These neural network models can be trained to detect diseases across a largely diverse species of plants and can grow proportional to the size of the dataset provided. In addition these models can be integrated in a mobile application and can be accessed without internetwork connectivity and the mobile application also displays suggestions of what pesticide to use, thus making it possible to use this system with very less computing power and in areas with poor or no network connections and hence enabling the use of technology in areas of agriculture and other flora based ecosystems on the go without much constraints.

\section{REFERENCES}

Ashqar, Belal and Abu-Naser, Samy. (2018). ImageBased Tomato Leaves Diseases Detection Using Deep Learning. International Journal of Academic Engineering Research. 2(12). 10-16

Ghulam Mustafa Choudhary and Vikrant Gulati (2015). Advance in Image Processing for Detection of Plant 
Diseases. International Journal of Advanced Research in Computer Science and Software Engineering 5(7), 1090-1093

Hrishikesh P. Kanjalkar, and S.S.Lokhande (2014). Feature Extraction of Leaf Diseases International Journal of Advanced Research in Computer Engineering \&t Technology. 3(1). 153-155

Image Processing and Support Vector Machine(SVM), Journal for Research, 02(02), 74 - 77.

Plant Leaf Disease Detection and Classification using Image Processing Techniques. International Journal of Innovative and Emerging Research in Engineering. 2(4) ,. 139-144.

PradnyaRavindra Narvekar, Mahesh Manik Kumbhar, \& S N Patil (2014). Grape Leaf Diseases Detection \& Analysis using SGDM Matrix Method. International Journal of Innovative Research in Computer and Communication Engineering. 2(3). 3365-3372. Prajakta Mitkal, Priyanka Pawar, Mira Nagane, Priyanka Bhosale, Mira Padwal and Priti Nagane (2016). Leaf Disease Detection and Prevention Using Image processing using MATLAB. 2(2), 26-30.

Prakash M. Mainkar, Shreekant Ghorpade, and Mayur
Adawadkar (2015).

Sabah Bashir and Navdeep Sharma (2012). Remote Area Plant Disease Detection using Image Processing. IOSR Journal of Electronics and Communication Engineering (IOSRJECE). 2(6), 31-34

Sen Maitra, Durjoy \& Bhattacharya, Ujjwal and Parui, Swapan. (2015). CNN based common approach to handwritten character recognition of multiple scripts. 1021-1025.

Sharada Prasanna Mohanty (2016). Using Deep Learning for Image-Based Plant Disease Detection. Frontiers in Plant Science, 1-10.

Sujatha R, Sravan Kumar Y and Garine Uma Akhil (2017). Leaf disease detection using image processing. Journal of Chemical and Pharmaceutical Sciences. 10(1), 670-672.

Vaijinath B Batule, Gaurav U Chavan, Vishal P Sanap and Kiran D Wadkar (2016), Leaf Disease Detection using

Vijai Singh,, A. K. Mishra (2017). Detection of plant leaf diseases using image segmentation and soft computing techniques. Information Processing In Agriculture, 4, 41-49. 